\title{
Эхо-картирование аккреционных дисков в активных ядрах галактик
}

\author{
С.Г. Сергеев \\ ФГБУН “Крымская астрофизическая обсерватория РАН”, Научный, Крым, 298409 \\ sergeev.crao@mail.ru
}

Поступила в редакцию 16 февраля 2018 г.

Аннотация. Представлен обзор работ по эхо-картированию аккреционных дисков в активных ядрах галактик (АЯГ). Метод эхо-картирования позволяет оценить ряд параметров этих дисков (в первую очередь размер диска) путем анализа многоволновой переменности АЯГ. Сделан вывод, что эхо-картирование аккреционных дисков работает, но есть проблемы, которые необходимо решать. Намечен план работ на будущее в этом направлении.

REVERBERATION MAPPING OF ACCRETION DISKS IN ACTIVE GALACTIC NUCLEI, by S.G. Sergeev. A review of papers on the reverberation mapping of accretion disks in active galactic nuclei (AGNs) is presented. The reverberation mapping method is used to obtain a number of parameters of these disks (primarily the disk size) by analyzing the multiwavelength variability of AGNs. It is concluded that the reverberation mapping of accretion disks works, but there are problems that need to be addressed. Plans for the future works in this direction are listed.

Ключевые слова: активные ядра галактик, сейфертовские галактики, аккреционные диски, переменность

\section{1 Введение}

Различные спектральные полосы, от рентгена до инфракрасной области в активных ядрах галактик (АЯГ), часто показывают коррелированные, хотя и не идентичные изменения потока. Это говорит о том, что некоторая доля первичной эмиссии из одного спектрального диапазона перерабатывается в другие диапазоны. Запаздывание по времени между наблюдаемыми кривыми блеска различных спектральных диапазонов может указывать на “первичный” диапазон, изменения потока которого инициируют изменения потока в других диапазонах.

Поскольку в рентгеновской области наблюдается самая большая и быстрая переменность потока, можно предположить, что рентгеновские лучи являются той самой первичной эмиссией. В рамках т. н. модели теплового репроцессинга (например, Коллин-Суфрин, 1991) аккреционный диск АЯГ излучает в оптическом и ультрафиолетовом диапазонах не только за счет механизма вязкого трения, но и за счет разогрева диска рентгеновским излучением. Если это так, то переменность оптического и ультрафиолетового излучения должна запаздывать относительно рентгеновского излучения и запаздывание должно быть тем больше, чем больше длина волны. Попытки измерения такого запаздывания (например, Користа и др., 1995; Колье и др., 1998, 1999; Шеммер и др., 2003; Сергеев и др., 
2005; Бридт и др., 2009) показали, что его величина (либо верхний предел) для разных АЯГ лежит в диапазоне от нескольких десятых дня до нескольких дней.

Для определения размера и других параметров аккреционного диска в рамках модели теплового репроцессинга может быть использован т. н. метод эхо-картирования (например, Блэндфорд, МакКи, 1982; Петерсон, 1993). Этот метод был разработан для определения размеров, геометрии и кинематики газа в области широких линий в АЯГ. При некоторых простых предположениях кривая блеска линии $L(t)$ связана с кривой блеска континуума $C(t)$ простым интегральным преобразованием, ядром которого является т. н. передаточная функция или функция отклика $\Psi(\tau)$, где $\tau>0$. Первый момент функции характеризует время запаздывания между $L(t)$ и $C(t)$. Поскольку нахождение этой функции из данных наблюдений, как правило, весьма проблематично, то для определения запаздывания между кривыми блеска используется кросс-корреляционная функция (например, Гаскелл, Спарк, 1986; Вайт, Петерсон, 1994). Данный метод с таким же успехом можно применить к аккреционным дискам. В этом случае запаздывание между кривыми блеска в различных спектральных диапазонах характеризует размер диска в этих диапазонах.

\section{2 Модель теплового репроцессинга}

В модели теплового репроцессинга оптическое и ультрафиолетовое излучение возникает, в основном, в аккреционном диске, который разогревается за счет рентгеновского излучения, источник которого горячая корона диска. Для простоты предполагается, что этот источник является точечным и расположен на высоте $h$ над центром диска (рис. 1). Поверхностная яркость участка диска на расстоянии

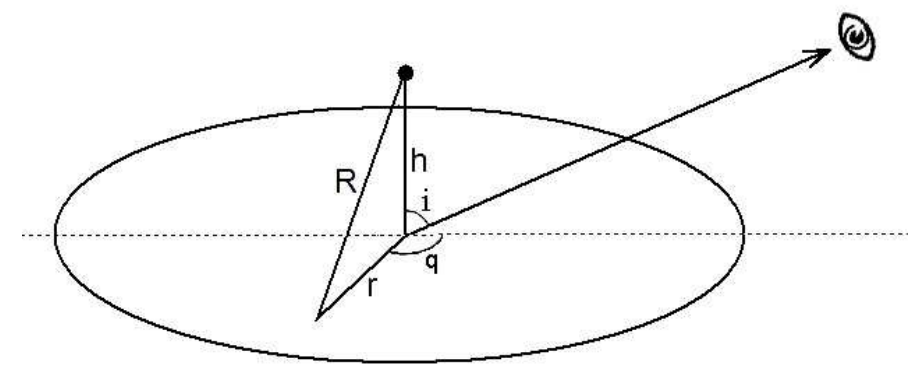

Рис. 1. Модель теплового излучения аккреционного диска. Здесь $i$ - угол наклона диска по отношению к лучу зрения, $h$ - высота источника рентгеновского излучения над диском, $r$ и $q$ - полярные координаты (расстояние и азимутальный угол). Рисунок взят из работы (Сергеев и др., 2005)

$r$ и при азимутальном угле $q$ равна $B(\lambda, T(r)) r d r d q$, где функция $B$ - это излучение абсолютно черного тела. Формула для расчета функции отклика приводится в нескольких работах. В работе (Сергеев и др., 2005) вывод этой формулы сделан следующим образом. Предполагая, что все рентгеновское излучение полностью перерабатывается в оптическое, мы имеем: $T^{4} \propto F_{X} \propto R^{-2}(h / R)$ и $T(r)=T_{0} h^{-1 / 4}\left(r^{2}+h^{2}\right)^{-3 / 8}$, где $T_{0}$ - температура на расстоянии 1 световой день от источника рентгеновского излучения, а $F_{X}$ - рентгеновский поток. Интегрируя по $r$, получаем наблюдаемый спектр $F(\lambda)$ на расстоянии $d$ от источника:

$$
F(\lambda)=\cos (i) \int 2 \pi r B(\lambda, T(r)) d r /\left(4 \pi d^{2}\right)
$$

Пересечение поверхностей равного запаздывания с поверхностью диска дает запаздывание $\tau$ как функцию $r$ и $q$ :

$$
\tau(r, q)=\left(r^{2}+h^{2}\right)^{1 / 2}+r \sin (i) \cos (q)+h \cos (i),
$$


и функция отклика вычисляется путем интегрирования по азимутальному углу из уравнения:

$$
\psi(\tau, \lambda) \propto \int r \frac{d r}{d \tau} B(\lambda, T(r)) d q,
$$

где $r$ и $\frac{d r}{d \tau}$ являются функциями $\tau$ и $q$, которые могут быть определены из (2), а область интегрирования для каждого $\tau$ определяется из геометрических соображений. Позже автором было замечено, что данное уравнение содержит некоторую неточность, поскольку связывает отклики потоков, а не изменения потоков.

Ожидаемая в рамках модели теплового репроцессинга зависимость запаздывания $\tau$ от длины волны $\lambda$ при $h \ll r$ следующая: $\tau \propto \lambda^{4 / 3}$.

\section{3 Обзор работ по эхо-картированию аккреционных дисков}

По-видимому, статистически значимое запаздывание между изменениями потоков в различных спектральных диапазонах (далее - межволновое запаздывание) было впервые обнаружено в работах (Колье и др., 1998, 1999). В этих работах были использованы спектральные данные ядра NGC 7469 и обнаружено запаздывание от ультрафиолетовых до оптических полос, которое зависело от длины волны, увеличиваясь в сторону более длинных волн (рис. 2). Это запаздывание

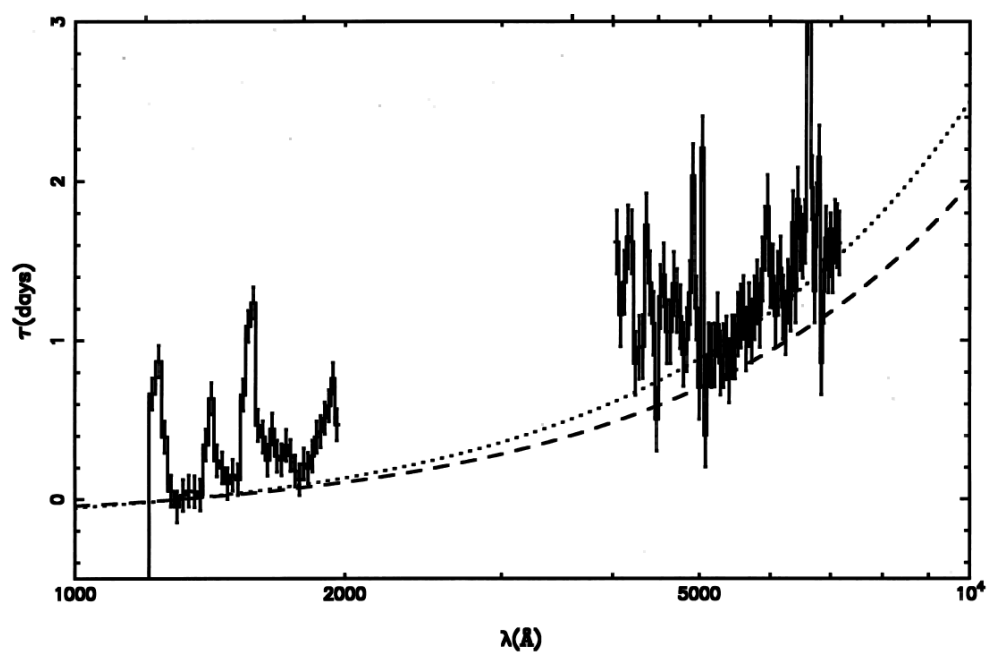

Рис. 2. Сравнение ожидаемых в рамках модели теплового репроцессинга запаздываний с наблюдаемыми запаздываниями, измеренными относительно $\lambda 1315 \AA$ для ядра NGC 7469. Время запаздывания отложено по вертикальной оси, а длина волны - по горизонтальной

было объяснено переработкой рентгеновских лучей в оптический диапазон в аккреционном диске. Моделируя наблюдаемый спектр и используя измеренные запаздывания, было получено независимое от красного смещения расстояние до NGC 7469, что позволило оценить постоянную Хаббла: $\mathrm{H}_{0}(\cos i / 0.7)^{1 / 2}=42 \pm 9 \mathrm{kм} \mathrm{c}^{-1} \mathrm{Mпc}^{-1}$, где $i-$ угол наклона аккреционного диска.

Впервые массовое измерение межволнового запаздывания (14 АЯГ) было сделано в работе (Сергеев и др., 2005) с использованием данных широкополосной фотометрии, полученных в КрАО. Было обнаружено, что:

- Имеется запаздывание между изменениями потока в фильтрах $V, R$ и $I$ по отношению к изменениям в фильтре $B$, которое составляет от долей дня до нескольких дней. 


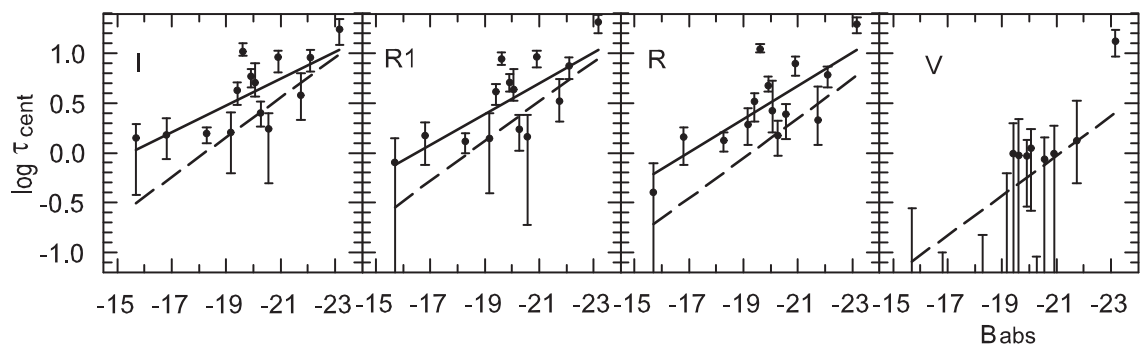

Рис. 3. Зависимость времени запаздывания в фотометрических полосах $V, R, R 1$ и $I$ по отношению к $B$ от абсолютной светимости для 14 АЯГ. Полоса, обозначенная как $R 1$, примерно соответствует фильтру $I_{c}$ системы Кузинса

- Это запаздывание тем больше, чем больше длина волны.

- Имеется зависимость между запаздыванием и абсолютной светимостью ядра: $\tau \propto L^{b}$, где $b \approx$ $0.4-0.5$ (рис. 3).

- В рамках модели теплового репроцессинга согласие с данными наблюдений может быть получено, если принять постоянную Хаббла равной $47 \mathrm{kм} \mathrm{с}^{-1} \mathrm{Mnc}^{-1}$.

В работе (Сергеев и др., 2005) указано, что вклад в фотометрические полосы излучения из более протяженных областей, таких как область широких линий, может искажать полученные результаты. Попытка учесть вклад этих линий в фотометрические полосы была сделана в работе (Сергеев, 2014), используя спектральные данные KрАО. Было показано, что в некоторых случаях такой учет может оказать заметное влияние на результаты измерения запаздывания.

Авторы работы (Бридт и др., 2009) использовали наблюдаемую рентгеновскую кривую блеска ядpa Mrk 79, чтобы в рамках модели теплового репроцессинга получить ожидаемую оптическую кривую блеска и сравнить ее с наблюдаемой кривой блеска. Параметрами модели являлись: высота источника рентгеновского излучения $h$, темп аккреции $\dot{m}$, угол наклона диска $i$ и внутренний радиус диска $R_{i n}$. Известная из других работ масса черной дыры этого ядра была использована для определения размера гравитационного радиуса черной дыры. На рис. 4 показана наблюдаемая оптическая кривая блеска вместе с подобранной наилучшим образом модельной кривой. Параметры этой модели равны: $h=21.5 R_{g}, i=24^{\circ}, \dot{m}=0.06$ (масс Солнца в год) и $R_{i n}=37.6 R_{g}$. Однако долговременный тренд в оптической области отсутствует в рентгеновском диапазоне и не может быть воспроизведен модельной оптической кривой, что хорошо видно из рис. 4. Было показано, что получить хорошее согласие между обеими кривыми можно при условии, что кратковременные изменения оптического потока являются следствием переработки рентгеновского потока в аккреционном диске, тогда как долговременные изменения (более тысячи дней) должны быть результатом независимого механизма переменности. Таким механизмом может являться долговременное изменение либо темпа аккреции, либо высоты источника рентгена.

Аналогичное моделирование для ядра NGC 4051 было сделано в работе (Бридт и др., 2010). Авторы этой работы обнаружили, что оптическая и рентгеновская кривые блеска показывают значительную корреляцию и что запаздывание между ними составляет $\tau=1.2_{-0.3}^{+1.0}$ дней (оптика запаздывает). Это запаздывание указывает на то, что оптические изменения происходят в результате переработки рентгеновских лучей. Однако оказалось, что такая переработка не может быть ответственна за всю оптическую переменность. Вероятно, за часть оптического излучения несет ответственность пылевой тор вокруг черной дыры. В пользу его существования свидетельствует вторичный пик в кросскорреляционной функции с запаздыванием $\tau=39$ дней. Это запаздывание согласуется с радиусом сублимации пыли в ядре NGC 4051. 


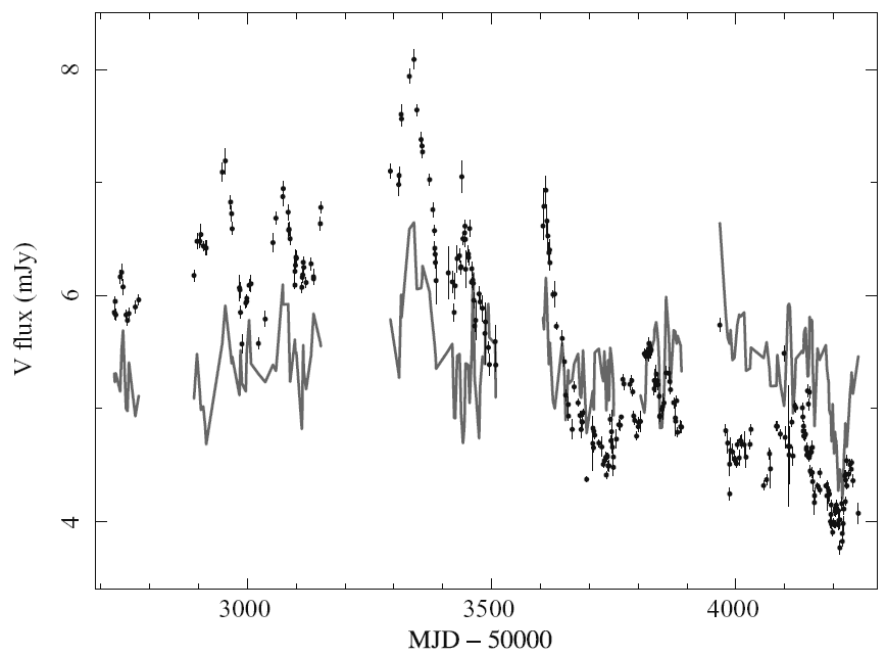

Рис. 4. Переменность наблюдаемого потока в полосе $V$ в ядре Mrk 79 (черные кружки с барами ошибок) не может быть воспроизведена из наблюдаемой рентгеновской кривой блеска в рамках простой модели теплового репроцессинга, где геометрия и скорость аккреции диска остаются неизменными. Модельная кривая блеска (сплошная линия) сильно отличается от наблюдаемой

В работе (Кэкит и др., 2007) сделана попытка воспроизвести, в рамках модели теплового репроцессинга, наблюдаемые запаздывания и спектральные распределения для 14 АЯГ по данным из работы (Сергеев и др., 2005). Эта модель дает для каждого объекта межзвездное покраснение его ядра, произведение массы черной дыры на темп аккреции и расстояния до объекта. Полученные расстояния не зависят от красного смещения и могут быть использованы как “стандартные свечи" для определения постоянной Хаббла, которая получилась равной $44 \pm 5$ км с $^{-1} M_{п c^{-1}}$.

Таблица 1. Запаздывания по отношению к полосе HST и максимальный коэффициент корреляции $\left(r_{\max }\right)$ для ядра NGC 5548

\begin{tabular}{|l|c|c|c|}
\hline Полоса & $\begin{array}{c}\text { Длина волны } \\
(\AA)\end{array}$ & $r_{\max }$ & $\begin{array}{c}\text { Запаздывание } \\
(\text { дней })\end{array}$ \\
\hline HX & 4.4 & 0.35 & $-0.66 \pm 0.46$ \\
SX & 25.3 & 0.44 & $+0.08 \pm 0.52$ \\
HST & 1367 & 1.00 & $+0.00 \pm 0.25$ \\
UVW2 & 1928 & 0.90 & $+0.40 \pm 0.17$ \\
UVM2 & 2246 & 0.87 & $+0.35 \pm 0.16$ \\
UVW1 & 2600 & 0.85 & $+0.61 \pm 0.20$ \\
U & 3465 & 0.81 & $+1.35 \pm 0.24$ \\
B & 4392 & 0.74 & $+1.23 \pm 0.29$ \\
V & 5468 & 0.57 & $+1.56 \pm 0.50$ \\
\hline
\end{tabular}

Авторы работы (Эдельсон и др., 2015) исследовали наблюдаемую переменность ядра NGC 5548 в девяти полосах от рентгена до оптики. Они обнаружили, что ультрафиолетовые и оптические полосы показывают значительную корреляцию с коэффициентом корреляции $r_{\max }=0.57-0.90$ при статистически значимых межволновых запаздываниях (более длинные волны запаздывают относительно более коротких). Найденные величины запаздываний (табл. 1) хорошо согласуются с зависимостью 
от длины волны $\tau \propto \lambda^{4 / 3}$, ожидаемой в рамках модели теплового репроцессинга, и дают неожиданно большой радиус диска $0.35 \pm 0.05$ св. дней на длине волны $\lambda 1367 \AA ̊$ в предположении, что диск виден плашмя.

Наиболее впечатляющие результаты в области эхо-картирования аккреционных дисков были получены в работе (Старки и др., 2017). Авторы этой работы провели анализ кривых блеска ядра NGC 5548 в 19 полосах от рентгена до ближней инфракрасной области. Переменность потока в ультрафиолетовой и оптической областях была ими интерпретирована как эхо-отклик аккреционного диска на точечный источник рентгена вблизи центра диска. На основании данных наблюдений была получена модель этого отклика, которая дает угол наклона диска $i=36^{\circ} \pm 10^{\circ}$, температуру диска $T_{1}=(44 \pm 6) \times 10^{3} \mathrm{~K}$ на расстоянии в 1 световой день от черной дыры и зависимость температуры от расстояния $T \propto r^{-\alpha}$, где $\alpha=0.99 \pm 0.03$. Данных наблюдений оказалось достаточно, чтобы в рамках этой модели была рассчитана первичная кривая блеска, которая вызывает переменность в других длинах волн и которой, как обычно считают, является рентгеновская кривая блеска. Однако рассчитанная первичная кривая блеска недостаточно хорошо коррелирует с наблюдаемой рентгеновской кривой блеска. Это указывает на то, что с помощью только одной рентгеновской переменности невозможно воспроизвести переменность на более длинных волнах.

\section{4 Планы нашего коллектива на будущее и проблемы}

1. Продолжение участия в международной программе STORM по эхо-картированию.

2. В духе работы (Сергеев и др., 2005) и других работ провести анализ богатого фотометрического материала, накопленного нашей группой в КрАО после 2005 г.

3. Решение имеющихся проблем и учет “подводных камней”. В частности, учет вклада широких линий в фотометрические кривые блеска. Необходимо также понять, почему только одна рентгеновская переменность не может объяснить переменности в ультрафиолете и оптике.

\section{5 Заключение}

1. Метод эхо-картирования и сопоставление кривых блеска в различных диапазонах длин волн в рамках модели теплового репроцессинга позволяют “увидеть” аккреционные диски АЯГ в ультрафиолетовом и оптическом диапазонах. В частности, можно получить такие параметры диска, как зависимость температуры от расстояния, угол наклона, темп аккреции, а также определить абсолютную светимость (стандартные свечи для космологии!).

2. Наряду с успехами картирования аккреционных дисков имеются проблемы и “подводные камни”.

\section{Литература}

Блэндфорд, МакКи (Blandford R.D., McKee C.F.) // Astrophys. J. 1982. V. 255. Р. 419.

Бридт и др. (Breedt E., Arévalo P., McHardy I.M., Uttley P., Sergeev S.G., et al.) // Mon. Not. Roy. Astron. Soc. 2009. V. 394. P. 427.

Бридт и др. (Breedt E., McHardy I.M., Arévalo P., Uttley P., Sergeev S.G., et al.) // Mon. Not. Roy. Astron. Soc. 2010. V. 403. P. 605.

Вайт, Петерсон (White R.J., Peterson B.M.) // Publ. Astron. Soc. Pacific. 1994. V. 106. P. 879.

Гаскелл, Спарк (Gaskell C.M., Sparke L.S.) // Astrophys. J. 1986. V. 305. P. 175.

Коллин-Суфрин (Collin-Souffrin S.) // Astron. Astrophys. 1991. V. 249. P. 344.

Колье и др. (Collier S.J., Horne K., Kaspi S., et al.) // Astrophys. J. 1998. V. 500. P. 162.

Колье и др. (Collier S., Horne K., Wanders I., Peterson B.M.) // Mon. Not. Roy. Astron. Soc. 1999. V. 302. P. L24.

Користа и др. (Korista K.T., Alloin D., Barr P., Clavel J., Cohen R.D., et al.) // Astrophys. J. Suppl. Ser. 1995. V. 97. P. 285. 
Кэкит и др. (Cackett E.M., Horne K., Winkler H.) // Mon. Not. Roy. Astron. Soc. 2007. V. 380. P. 669. Петерсон (Peterson B.M.) // Publ. Astron. Soc. Pacific. 1993. V. 105. P. 247.

Сергеев и др. (Sergeev S.G., Doroshenko V.T., Golubinskiy Yu.V., Merkulova N.I., Sergeeva E.A.) // Astrophys. J. 2005. V. 622. P. 129.

Сергеев (Sergeev S.G.) // Multiwavelength AGN Surveys and Studies. IAU Symp. N. 304. / Eds Mickaelian A., Sanders D. Cambridge: Cambridge University Press. 2014. P. 247.

Старки и др. (Starkey D., Horne K., Fausnaugh M.M., et al.) // Astrophys. J. 2017. V. 835. P. 65.

Шеммер и др. (Shemmer O., Uttley P., Netzer H., McHardy I.M.) // Mon. Not. Roy. Astron. Soc. 2003. V. 343. P. 1341.

Эдельсон и др. (Edelson R., Gelbord J. M., Horne K., et al.) // Astrophys. J. 2015. V. 806. Р. 129. 